\title{
Utilization of Cellulose from Luffa cylindrica Fiber as Binder in Acetaminophen Tablets
}

\author{
John Carlo O. Macuja, ${ }^{1}$ Laurence N. Ruedas, ${ }^{1}$ and Rebecca C. Nueva España ${ }^{2}$ \\ ${ }^{1}$ Faculty of Science, Technology and Mathematics, College of Teacher Development, Philippine Normal University, \\ 1000 Manila, Philippines \\ ${ }^{2}$ College of Graduate Studies and Teacher Education Research, Philippine Normal University, 1000 Manila, Philippines
}

Correspondence should be addressed to John Carlo O. Macuja; jcomacuja@gmail.com

Received 27 September 2014; Revised 22 February 2015; Accepted 22 February 2015

Academic Editor: Jesus Simal-Gandara

\begin{abstract}
Copyright (C) 2015 John Carlo O. Macuja et al. This is an open access article distributed under the Creative Commons Attribution License, which permits unrestricted use, distribution, and reproduction in any medium, provided the original work is properly cited.
\end{abstract}

Cellulose is an important pharmaceutical excipient. This study aimed to produce cellulose from the fiber of Luffa cylindrica as an effective binder in the formulation of acetaminophen tablets. This study was divided into three phases, namely, (I) preparation of cellulose from Luffa cylindrica, (II) determination of the powder properties of the LC-cellulose, and (III) production and evaluation of acetaminophen of the tablets produced using LC-cellulose as binder. The percentage yield of LC-cellulose was $61 \%$. The values of the powder properties of LC-cellulose produced show fair and passable flow properties and are within the specifications of a powdered pharmaceutical excipient. The mean tablet hardness and disintegration time of the LC-cellulose tablets have a significant difference in the mean tablet hardness and disintegration time of the tablets without binder; thus the cellulose produced improved the suitability of acetaminophen in the dry compression process. However, the tablet properties of the tablets produced using LCcellulose as binder do not conform to the specifications of the US pharmacopeia; thus the study of additional methods and excipients is recommended.

\section{Introduction}

Microcrystalline cellulose (MCC) is purified and partially depolymerized alpha cellulose derived from the pulp of fibrous plants. MCC occurs as a white, odorless, tasteless, and crystalline powder composed of porous particles. The common sources of commercial MCC are wood pulp and purified cotton linters. MCC can be made from any material that is high in cellulose ranging from pure cellulose, commercial grade cellulose to lignocellulosic materials [1].

Microcrystalline cellulose is widely used in the pharmaceutical industry. It is commonly used as an excipient in tablet formulations. Excipients are pharmacologically inactive substances that are formulated with the active ingredient in drugs. Most common types of excipients include binders. Apart from being physiologically inert, MCC is an excellent excipient because of its compressibility in solid dosage forms. Microcrystalline cellulose is mostly used as filler binder in both wet or dry formulation and direct tablet compression.
Its popularity in direct compression is due to its excellent binding properties when used as a dry binder [2]. MCC also has disintegrant and lubricant properties. It combines two useful properties of tablet vehicle; it can produce very hard tablets and yet these tablets disintegrate rapidly in water due to swelling of the MCC particles and destruction of the bonding forces holding them together.

Luffa cylindrica fibers contain about $60-63 \%$ alpha cellulose. This makes Luffa cylindrica one of the good sources of microcrystalline cellulose. Luffa cylindrica fiber is investigated to be an alternative source of MCC. A major source of MCC such as cotton is a high-value added crop. Wood pulp on the other hand originates in some manner from deforestation which is not environment friendly.

The purpose of this study is to utilize the cellulose from Luffa cylindrica as an effective binding agent in the production of acetaminophen tablets. This study reports the powder properties of the obtained cellulose specifically: bulk density, tapped density, and angle of repose. The properties of the 


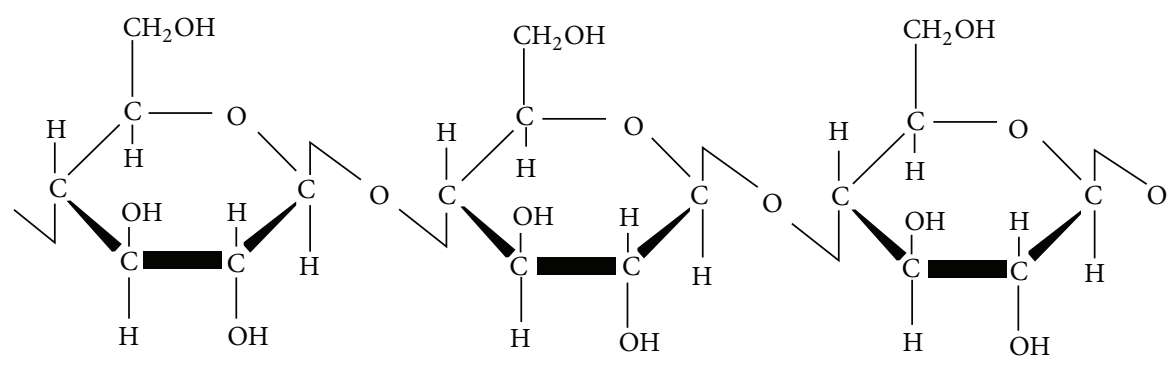

FIGURE 1: Structure of cellulose.

tablets produced such as tablet hardness and disintegration time are also reported.

Cellulose. Cellulose is the most abundant organic polymer in the world today. It is a linear polysaccharide consisting of $B$ $D$-Glucose units linked by $ß(1 \rightarrow 4)$ glycosidic bonds. It is the major structural component of the rigid cell walls in plants. Cellulose is mostly present in wood, cotton, and plant fibers. The structure of cellulose is shown in Figure 1.

In 1838, Anselme Payne discovered cellulose by isolating it in a plant material. He was also the one who determined its molecular formula. Cellulose is a major constituent of paper, paper board, and textiles. Pure cellulose or alpha cellulose can be obtained from wood pulp and plant fibers by treating it with sodium hydroxide to separate lignin from it. When $\alpha$-cellulose is then treated with a dilute mineral acid, a new product is then formed. This product is called microcrystalline cellulose (MCC).

Microcrystalline Cellulose. Microcrystalline cellulose (MCC) is the purified and partially depolymerized alpha cellulose derived from the pulp of fibrous plants. In treating $\alpha$-cellulose with a dilute mineral acid, the acid penetrates the amorphous regions relatively fast and hydrolyzes it yielding water-soluble fragments or oligomers. The time required for hydrolysis to take place is just sufficient such that it will not disrupt the crystalline regions leaving those segments intact.

MCC occurs as a white, odorless, tasteless, and crystalline powder composed of porous particles. Woody plants and cotton are the major sources of MCC but cost has made it imperative that other materials rich in cellulose be investigated as potential sources. MCC can be made from any material that is high in cellulose ranging from pure cellulose, commercial grade cellulose to lignocellulosic materials [1]. Plant materials that are potential sources of microcrystalline cellulose include soy bean husk, oat and rice hulls, sugar beet pulp, groundnut shell and rice husks, straws of wheat, barley, oat and cereals, Indian bamboo, and reed stalks. Agricultural waste materials such as bagasse and corn cob can also be utilized as potential sources of MCC.

Uses of Microcrystalline Cellulose. Microcrystalline cellulose is widely used in the pharmaceutical, food, and cosmetics industries. In the pharmaceutical industry, MCC is an important ingredient as an excipient in the formulation of tablets. Excipients are pharmacologically inactive substances that are formulated with the active ingredient in drugs. It is commonly used to bulk up formulations that contain potent active ingredients to allow convenient and accurate dispensation of a drug substance when producing a dosage form. It facilitates drug absorption or solubility, or other pharmacokinetic considerations.

In the study of Ogaji et al. [3] excipients are defined as substances, other than the active drug of finished dosage form, which have been appropriately evaluated for safety and are included in a drug delivery system to either aid the processing of the drug delivery system during its manufacture, protect, support, or enhance stability, bioavailability, or patient acceptability. Excipients play a very important role in the production of medicine tablets. They help preserve the efficacy, safety, and stability of active pharmaceutical ingredients (APIs) and they ensure that the drug delivers its promised benefits to the patients.

Optimal use of excipients can provide pharmaceutical manufacturers with cost savings in drug development, enhanced functionality, and help in drug formulations innovation [3]. Types of excipients used in tablet formulations include fillers/diluents, binders, disintegrants, lubricants, glidants, and miscellaneous. Fillers increase the volume or bulk content of the dosage form; this is done in a situation where the active constituent to be incorporated in the formulation is of less quantity. Binders hold the ingredients in a tablet together. It ensures that tablets and granules can be formed with required mechanical strength. Disintegrants are a type of excipient that expand and dissolve when wet causing the tablet to break apart in the digestive tract, releasing the active ingredients for absorption. Disintegrants facilitates the dissolution of the tablets. Lubricants and glidants almost have similar function. Lubricants reduce friction between the tablet and mold of the tablet. Glidants on the other hand are used to promote powder flow by reducing interparticle friction and cohesion. Miscellaneous excipients include flavorants and coloring agents.

The crucial role and application of natural polymeric materials such as microcrystalline cellulose in the pharmaceutical industry is attributed to the fact that it is a biodegradable and toxicologically harmless raw material of low cost and relative abundance. Natural polysaccharides such as MCC are also derived from natural resources that are renewable and can be cultivated or harvested in a sustainable manner. This can provide a constant supply of raw material for the production of commercial MCC. Furthermore, their 
wide-ranging applications in drug delivery have been realized because, as polymers, they offer distinctive properties which so far have not been attained by any other materials. They can also be modified for many applications based on the very large chains and functional groups which can be blended with other low- and high-molecular-weight materials to achieve novel materials with various physicochemical properties.

Other uses of microcrystalline cellulose are seen in the food and cosmetics industries. MCC is used as anticaking agent, emulsifying agent, fat substitute or replacer, bulking agent, and stabilizer and texturizing agent in food production. Microcrystalline cellulose gels are used as emulsion stabilizers, thickeners, and viscosity regulators in pastes, creams, and lotions [4]. Its hydrocolloid compatibility makes it useful in the manufacture of creams, lotions, and various cosmetics emulsion.

Tablet Binders. Tablets are the most commonly used pharmaceutical dosage form. It consists of a mixture of the active pharmaceutical ingredients and excipients. Tablets are usually in powder form, pressed, or compacted to form into a solid dose. The ease of manufacturing, convenience in administration, accurate dosing, stability, tamper proofness, and safety make tablets a popular and versatile dosage form. In the tablet-making process, it is important that all ingredients are fairly dry, powdered or granular, somewhat uniform in particle size, and freely flowing. The role of tablet binding agents is crucial in this part of the manufacturing process. Binders are the substances responsible for holding the active ingredients and excipients together.

Binders are agents used to impart cohesive qualities to the powdered material during the production of tablets. Binders ensure that the tablet remains intact after compression; in addition, it also improves its free-flowing quality. Binders are added to tablet formulation to impart plasticity and thus increase the interparticulate bonding strength within the tablet [5]. According to the study of Gaikwad and Kulkarni [6], binders are used as solutions and in dry form depending on the other ingredients in the formulations and method of preparation. The choice of a specific binding agent depends on the binding force required to form granules and its compatibility with the other ingredients, especially the drug or the active component of the mixture. Binders can be classified according to their source and method of application.

There are three classifications of binders according to their source, namely, natural polymers, synthetic polymers, and sugars. Natural polymers include polysaccharides such as starch, cellulose and modified cellulose (microcrystalline cellulose), gelatin, acacia, and gums. Synthetic polymers include polyvinyl pyrrolidone, HPMC, methyl cellulose, ethyl cellulose, and polyethylene glycol. Sugar binders include lactose, sucrose, xylitol, and sorbitol. Based on the method of application, binders can be either solution binder or dry binder. Solution binders are dissolved in a solvent (e.g., water or alcohol can be used in wet granulation processes). Examples of solution binders are gelatin, cellulose, cellulose derivatives, polyvinyl pyrrolidone, starch, sucrose, and polyethylene glycol. Dry binders on the other hand are added to the powder blend, either after a wet granulation step or as part of a direct powder compression (DC) formula. Examples include cellulose, methyl cellulose, polyvinylpyrrolidone, and polyethylene glycol. Natural binders are preferred in the pharmaceutical industry because of their advantages.

Natural polysaccharides are widely used in the pharmaceutical and food industries as excipients and additives because they are physiologically inert, biodegradable, readily available, and with low cost. Another advantage of them is that they can also be used to modify the release of drug, by this means, influencing the absorption and subsequent bioavailability of the incorporated drug. Natural binders act as vehicles which transport the incorporated drug to the site of absorption and are expected to guarantee the stability of the incorporated drug, the precision and accuracy of the dosage, and also improve the organoleptic properties of the drugs which is necessary in order to enhance patient adherence. They also optimize the performances of dosage forms during manufacturing as well as when patients ingest them.

Several studies have been conducted on natural binders for tablet formulations. In the study of Shailendra et al. [5], several natural binders are listed. This includes natural polysaccharides such as tapioca starch, rice starch, corn starch, potato starch, wheat starch, Manihot esculenta or cassava starch [7], and Hordeum vulgare or barley starch [8]. Natural gums such as Ferula gummosa Boiss, gum olibanum, Beilschmiedia seed gum, Okra gum, Aegle marmelos gum, and Cassia roxburghii seed gum are also potential binding agents in tablet formulations.

Luffa cylindrica. Luffa cylindrica commonly known as "Patola" or "Patolang bilog" is a tropical plant belonging to the family Cucurbitacea. This plant is a climbing, hairy, and smooth vine with a maximum length of 12 meters. The fruit of Luffa cylindrica is cylindric, smooth, and green with a length of 12 to 30 centimeters.

The fruit also has a netting-like fibrous vascular system. One of the most important uses of Luffa cylindrica is for bath sponges. The fully ripened fruit is fibrous and becomes the source of loofah scrubbing sponge which is used in bathrooms and kitchens as a sponge tool.

Luffa cylindrica fiber is a source of lignocellulosic materials. It contains cellulose, hemicelluloses, and lignin. In the study of Siqueira et al. [9], Luffa cylindrica fibers contain about $10.6-11.2 \%$ lignin, 19.4-22\% hemicelluloses, and 60$63 \%$ alpha cellulose. In this study Luffa cylindrica fibers had been investigated as source of microfibrillated cellulose and cellulose nanocrystals. MFC consists of high aspect ratio cellulose nanofibrils prepared by mechanical homogenization of cellulose fibers. Cellulose nanocrystals also called whiskers are high purity single cellulose crystals obtained after treatment of fibers under controlled conditions. Cellulose nanocrystals can be used in the preparation of nanocomposites with increased strength and extraordinary properties.

Aside from being a lignocellulosic source of fiber, several studies have shown that Luffa cylindrica contains different phytochemicals that have a significant activity. In the study of Arunachalam et al. [10], preliminary phytochemical screening confirmed the presence of carbohydrates, flavanoids, glycosides, and saponins. Chloroform extract of the whole 
plant of Luffa cylindrica (Linn) was also found to have significant antibacterial and antifungal activity [11]. Several studies also show that the seeds of the Luffa plant have important uses. In the study of Yoganandam et al. [12], the oil obtained from L. cylindrica had shown significant antioxidant activity. DPPH free radical, nitric oxide radical, and hydroxyl radical scavenging assays were carried out to evaluate the in vitro antioxidant activity of Luffa cylindrica seed oil. The antioxidant potential may be attributed to the presence of polyunsaturated compounds specifically stearic and linoleic acid. The extracts and isolated compounds from the seed oil of Luffa cylindrica were also found to have anti-inflammatory and bronchodilator activity [13]. When it comes to biodiesel properties Luffa cylindrica oil and its ethyl ester were found to have good combustion characteristics and good ignition quality. Blends of Luffa ethyl ester were found to have acceptable fuel properties to power compression ignition engines [14].

Related Studies to Microcrystalline Cellulose. In the study of Ohwoavworhua and Adelakun [15], microcrystalline cellulose was obtained from the raw cotton of Cochlospermum planchonii. Cochlospermum planchonii is a low shrubby savanna plant cultivated in the savanna zones of Guinea and Sudan. The plant produces 3-5 celled capsules containing seeds whose epidermal trichomes are cotton. The percentage yield of microcrystalline cellulose from the cotton material was $21 \% \mathrm{w} / \mathrm{w}$. The powder properties of MCC obtained from the cotton were examined and compared with the commercial grade microcrystalline cellulose which is Avicel PH 101. When compared with the powder properties of Avicel PH 101 results show that the MCC from Cochlospermum planchonii has poor flow properties and would require the addition of a glidant during the tableting process. On the other hand the swelling parameters indicate that CP-MCC would be a better disintegrant than the standard MCC. The MCC obtained still conformed to the official specifications of the British pharmacopoeia (2004).

Agricultural waste lignocellulosic materials were also studied as potential sources of low cost microcrystalline cellulose. One agricultural waste product rich in cellulose is sawdust. Sawdust is obtained in the process of smoothing timber. In the study of Oyeniyi and Itiola [16] the percentage yield of MCC from sawdust was $68 \% \mathrm{w} / \mathrm{w}$. This indicates that sawdust is a very good source of alpha cellulose. Particle size analysis of the MCC produced from sawdust reveals that the average particle size is $62 \mu \mathrm{m}$. This falls in the $60-70 \mu \mathrm{m}$ range of particle size of the commercial MCCs. Results of the study also show that the powder properties of the SD-MCC and Avicel PH 101 are similar and both have excellent flow properties. The angle of repose of the sawdust MCC is $26^{\circ}$. This angle is within the range of values that is considered as excellent $\left(25-39^{\circ}\right)$.

A nonwood fiber source of MCC and another agricultural waste is orange mesocarp. Ejikeme [1] in his study investigated the physicochemical properties of the MCC produced from orange mesocarp. Results obtained from the study showed that the percentage yield of alpha cellulose from orange mesocarp is $62.5 \%$. The percentage yield of MCC on the other hand is $25.3 \% \mathrm{w} / \mathrm{w}$. The MCC obtained from orange mesocarp is soluble only in the ammoniacal solution of copper tetrammine. The $\mathrm{pH}$ and moisture content of the MCC produced are 6.61 and $4.9 \%$, respectively. These values were within the limits specified by the British pharmacopoeia, 5-7 for $\mathrm{pH}$ and up to $8 \%$ for moisture content. The MCC obtained from orange mesocarp was also free from impurities since it yielded negative results in the test for starch and dextrin. It also gave a blue-violet color in the identification test for MCC.

In the research conducted by Azubuike and Okhamafe [17], microcrystalline cellulose was derived from corn cobs. The spectroscopic, thermal, and physicochemical properties of the derived $\alpha$-cellulose and microcrystalline cellulose powders were compared with Avicel PH 101, a commercial brand of microcrystalline cellulose (MCCA), using standard methods. Studies of the X-ray diffraction of the samples showed that the microcrystalline cellulose samples obtained from maize cobs had diffraction pattern characteristics of both cellulose I and cellulose II, whereas Avicel PH 101 had that of cellulose I; however, all the microcrystalline cellulose samples had similar crystallinity index (CI) values. Infrared spectroscopy results showed that the microcrystalline cellulose samples had comparable CI values and molecular structure.

Several studies have been conducted on microcrystalline cellulose as binder, diluent or filler and disintegrant. In the study of Bastos et al. [18], entitled "Effects of Filler-Binders and Lubricants on Physicochemical Properties of Tablets Obtained by Direct Compression," the influence of the fillerbinder granulometry on the physicochemical characteristics of tablets containing high amount of an active substance has been investigated. The MCC samples used are Avicel PH 101 and Avicel PH 102 which are the standard commercial MCCs. Results of the study showed that the filler-binder granulometry of the MCC has a significant influence on the tablet properties such as hardness and friability. Formulations containing Avicel $\mathrm{PH}-102$ presented higher hardness and lower friability as compared with those prepared with Avicel $\mathrm{PH}-101$. The higher hardness of the tablets formulated using Avicel PH 102 is attributed to its longer surface area and higher granulometry.

Microcrystalline cellulose can also be used as a disintegrant in tablet formulations. In the study of Chowdary et al. [19] microcrystalline cellulose was used as a disintegrant in Lomefloxacin tablets. Lomefloxacin is a broad spectrum antibacterial agent used in the treatment of respiratory tract and urinary tract infections. The problem with this drug is its dissolution and bioavailability in the biological fluids after oral administration. The study was aimed at evaluating the effects of formulation variables such as binding agents and disintegrants on the dissolution rate of the antibiotic. When it comes to MCC, results of the study showed that when MCC was used as disintegrant, the tablet disintegrates rapidly as compared with the two disintegrants like dry potato starch and sodium starch glycolate.

In the study of Gaikwad and Kulkarni [6], microcrystalline cellulose was used as a diluent in the development of paracetamol tablets using Buchanania cochinchinensis gum as binder. The effects of different diluents and disintegrants were also studied using optimized concentration of the 
gum. Focusing on the effect of microcrystalline cellulose as diluents, results showed that microcrystalline cellulose showed slightly higher drug release compared with the two other diluents which are lactose and dicalcium phosphate.

\section{Materials and Methods}

This study is divided into three phases, namely, (I) preparation of MCC from Luffa cylindrica, (II) determination of the physicochemical and powder properties of the LC-MCC, and (III) production and evaluation acetaminophen of the tablets produced using LC-MCC as binder.

\section{Materials}

List of Reagents. The used reagents were acetaminophen, distilled water, hydrochloric acid, magnesium stearate, sodium hydroxide, and sodium hypochlorite.

List of Apparatus. Apparatus used were analytical balance, beakers, graduated cylinder, hot plate, laboratory mill, oven, short stem funnel, stirring rod, and thermometer.

\subsection{Phase I: Preparation of MCC from Luffa cylindrica}

2.1.1. Collection of Raw Materials. The ripened fruits containing the fiber of Luffa cylindrica were collected and authenticated at the Bureau of Plant Industry. The skin of the Luffa cylindrica was peeled off and fiber inside was washed using running water and sun dried for 3 days. The seeds and other impurities were separated manually by shaking to let the seeds fall; other seeds that clung to the fiber were plucked. The collected fibers were reduced into smaller pieces using Wiley laboratory mill in the pharmaceutical section of the Department of Science and Technology.

2.1.2. Isolation of $\alpha$-Cellulose. The Luffa cylindrica fiber was treated with $4 \%(\mathrm{w} / \mathrm{v})$ sodium hydroxide for 180 minutes at $80^{\circ} \mathrm{C}$ in a water bath. After thorough washing and filtration, it was then bleached with $5.3 \%(\mathrm{w} / \mathrm{v})$ sodium hypochlorite for 15 minutes at $80^{\circ} \mathrm{C}$. The sample was then washed with distilled water and was treated with $17.5 \%(\mathrm{w} / \mathrm{v})$ sodium hydroxide at $80^{\circ} \mathrm{C}$ for $1 \mathrm{hr}$. The extraction process was completed by bleaching it again with sodium hypochlorite for 15 minutes. The product will then be thoroughly washed and oven dried for 25 minutes.

2.1.3. Production of MCC. The obtained alpha cellulose was hydrolyzed using $2.5 \mathrm{M}$ hydrochloric acid at a temperature of $105^{\circ} \mathrm{C}$ for 15 minutes. The MCC collected was filtered and washed with deionized water until neutral. It was then oven dried for 15 minutes and stored in an air tight desiccator. The percentage yield of MCC was computed using the formula

Percent Yield of MCC $(\%)=\frac{A}{B} \times 100$,

$A(\mathrm{mg})=$ Weight of obtained microcrystalline cellulose,

$B(\mathrm{mg})=$ Weight of alpha cellulose.

\subsection{Phase II: Determination of Powder Properties of LC-MCC}

\subsubsection{Powder Properties}

Bulk and Tapped Densities. In the determination of the bulk density, $20 \mathrm{~g}$ of MCC produced was placed in a $100 \mathrm{~mL}$ clean and dry cylinder. The bulk volume, $V_{0}$, occupied by the sample was recorded. For tapped density, the graduated cylinder containing $20 \mathrm{~g}$ of the sample was tapped from a height of $2.5 \mathrm{~cm}$ until constant volume was reached. The tapped volume, $V_{T}$, occupied was recorded. The bulk and tap densities were calculated as the ratio of weight to volume $\left(V_{0}\right.$ and $V_{T}$, resp.).

Carr's Index. Carr's index or the compressibility index of the MCC was calculated using

$$
\text { Carr's index }=\frac{D_{\text {tap }}-D_{\text {bulk }}}{D_{\text {tap }}} \times 100 .
$$

Hausner Ratio. Hausner ratio was calculated using

$$
\text { Hausner ratio }=\frac{D_{\text {tap }}}{D_{\text {bulk }}} .
$$

Angle of Repose. The static angle of repose was determined using the fixed funnel and free standing cone method. A funnel was clamped with its tip $2 \mathrm{~cm}$ above a graph paper raised on a horizontal surface. The sample powder was poured through the funnel until the apex of the cone formed reached the tip of the funnel. The height $(h)$ and radius $(r)$ of the cone were recorded and the angle of repose was calculated using

$$
\theta=\tan ^{-1}\left(\frac{h}{r}\right) .
$$

\subsection{Phase III: Evaluation of the Tablet Properties of the Produced LC-MCC}

2.3.1. Tablet Formulation and Compression. The acetaminophen tablets were formulated and produced at the University of Philippines Manila, College of Industrial Pharmacy. The tablet formulation consisted of $91 \%$ acetaminophen and $6 \%$ binder and 3\% lubricant. The total weight of each tablet made was $500 \mathrm{mg}$. Acetaminophen tablets were made by mixing the acetaminophen powder and the produced MCC as binder. The resulting mixture was sieved using mesh screen \#60 to produce a fine powder. The sieved mixture was then lubricated with magnesium stearate. The final mixture was subjected to mechanical tablet compressor for dry compression. One batch of tablets was made using the LC-MCC as a binder and another batch of acetaminophen tablets was compressed without binder. The weight and thickness of the produced acetaminophen tablets were noted.

2.3.2. Tablet Strength Analysis. The hardness or the strength of the tablets produced from the two batches was then determined using the Stoke tablet hardness tester in the College of Industrial Pharmacy at University of the Philippines. The values were compared and statistically analyzed using the $t$ test for independent means. 
TABLE 1: Mass of obtained alpha cellulose and produced LC-MCC.

\begin{tabular}{lc}
\hline Powder & Mass (g) \\
\hline Alpha cellulose & 29.85 \\
LC-MCC & 18.115 \\
\hline
\end{tabular}

TABLE 2: Bulk and tapped density of LC-MCC produced.

\begin{tabular}{lcc}
\hline Trial & Bulk density & Tapped density \\
\hline 1 & 0.231 & 0.290 \\
2 & 0.241 & 0.308 \\
3 & 0.222 & 0.282 \\
\hline Average & 0.231 & 0.293 \\
\hline
\end{tabular}

TABLE 3: Powder properties of LC-MCC produced.

\begin{tabular}{lcc}
\hline Powder property & Value & Remarks \\
\hline Carr's index & $21 \%$ & Passable \\
Hausner index & 1.27 & Passable \\
Angle of repose & $37^{\circ}$ & Fair \\
\hline
\end{tabular}

2.3.3. Tablet Disintegration. The disintegration time of acetaminophen tablets was determined using tablet disintegrator machine available at University of the Philippines, College of Industrial Pharmacy. The procedure for the determination of tablet disintegration time was based on the US pharmacopeia.

\section{Results and Discussion}

This section presents all of data gathered in the study that will answer the statement of the problem. The results of the following are reported in this section: percentage yield of microcrystalline cellulose from Luffa cylindrica fiber, powder properties of LC-MCC, tablet hardness, and disintegration time of acetaminophen tablets produced using LC-MCC as binder.

Using (1) and from Table 1 we can get the following:

$$
\begin{aligned}
\text { Percentage Yield of MCC }(\%) & =\frac{18.115}{29.85} \times 100 \\
& =60.69 \% .
\end{aligned}
$$

The percentage yield of microcrystalline cellulose from the fiber of Luffa cylindrical was approximately $60.69 \%$ or $61 \%$. This suggests a high percentage yield compared with the $21 \%$ extraction yield of MCC in the raw cotton of Cochlospermum planchonii [2] and $25.3 \%$ in orange mesocarp [1]. The $60.69 \%$ percentage yield of MCC from the fiber of Luffa cylindrica is relatively high enough to stimulate large scale commercialization of the extraction of MCC. 3.
Carr's Index. Carr's index or the compressibility index of the MCC was calculated using (2):

$$
\begin{aligned}
\text { Carr's index } & =\frac{0.293-0.231}{0.293} \times 100 \\
& =21 \% .
\end{aligned}
$$

Hausner Ratio. Hausner ratio was calculated using (3):

$$
\begin{aligned}
\text { Hausner ratio } & =\frac{0.293}{0.231} \\
& =1.27
\end{aligned}
$$

Angle of Repose. Consider the following:

$$
\theta=37.04^{\circ}
$$

The calculated values for the powder properties of the produced microcrystalline cellulose indicate that the LCMCC has fair and passable flow properties. The flow properties of a powdered material (Carr's compressibility index, Hausner index, and angle of repose) are essential in determining its suitability as a direct compression excipient [2]. These properties are considered as indirect measurements of powder flowability. Carr's index gives an idea of how much a powder can be compressed; it also shows the aptitude of material to diminish in volume. The computed Carr's index was $21 \%$. This value is within the passable mark of Carr's scale of flowability. This also suggests good flowability because it does not exceed $35 \%$ which corresponds to very poor flow. The calculated Hausner index on the other hand is 1.27 which also corresponds to the passable range of Carr's scale of flowability. The Hausner index or ratio measures cohesion between particles.

The angle of repose is the constant three-dimensional angle assumed by a cone-like pile of material. It is a characteristic related to interparticulate friction or resistance to movement between particles. The angle of repose measured from the microcrystalline cellulose produced from Luffa cylindrica was 37 degrees. This value falls within the remark of fair in Carr's scale of flowability. The value of the angle of repose also indicates good flow because it does not exceed the limit of 45 degrees which corresponds to very poor flow. The LC-MCC angle of repose is also within the specification of the US pharmacopoeia 2000. Pharmaceutical powders with angle of repose value 25-39 degrees are considered excellent [16].

3.2. Tablet Properties. Table 4 shows the values of the hardness of the 2 batches of tablets produced. One batch is formulated using LC-MCC as binder and the other batch is formulated and compressed without binder. The data shows that tablets produced using LC-MCC as binder have higher hardness compared with the tablets produced without binder. Thus it improved the suitability of acetaminophen in the dry compression process.

Results of the statistical analysis used in Table 5 show that the mean tablet strength of the MCC tablets has a significant difference in the mean hardness of the tablets 
TABLE 4: Hardness of acetaminophen tablets produced.

\begin{tabular}{lcc}
\hline Trial & $\begin{array}{c}\text { MCC tablet hardness } \\
(\mathrm{N})\end{array}$ & $\begin{array}{c}\text { Tablet w/o binder } \\
\text { hardness }(\mathrm{N})\end{array}$ \\
\hline 1 & 2 & 1 \\
2 & 2 & 1.1 \\
3 & 2.5 & 0.8 \\
\hline Average & 2.17 & 0.97 \\
\hline
\end{tabular}

TABLE 5: Data for $t$-test for independent means.

\begin{tabular}{lcc}
\hline & MCC tablet & w/o binder \\
\hline Mean & 2.17 & 0.97 \\
Variance & 0.083 & 0.023 \\
Observations & 3 & 3 \\
Pooled variance & 0.053 & \\
Hypothesized mean difference & 0 & \\
df & 4 & \\
$t$ stat & $\mathbf{6 . 3 6 4}$ & \\
$P(T<=t)$ one-tail & 0.0016 & \\
$t$ critical one-tail & 2.132 & \\
$P(T<=t)$ two-tail & $\mathbf{0 . 0 0 3}$ & \\
$t$ critical two-tail & $\mathbf{2 . 7 7 6}$ & \\
\hline
\end{tabular}

$t$-test: two-sample test assuming equal variances.

TABLE 6: Disintegration time of tablets produced.

\begin{tabular}{lcc}
\hline Trial & MCC $($ mins $)$ & w/o binder $(\mathrm{mins})$ \\
\hline 1 & 29.38 & 0.33 \\
2 & 34.24 & 0.92 \\
3 & 35.1 & 5.37 \\
\hline Average & 32.91 & 2.21 \\
\hline
\end{tabular}

produced without binder. The $P$ value of 0.003 is less than the confidence level of 0.05 ; thus the data has a significant difference. The test statistic value of 6.36 is also greater than the critical value of $t$ (2.776) which also indicates that there is a significant difference in the mean hardness of the two types of tablets. However, based on standards given by the United States pharmacopeia, the acceptable hardness for commercial tablets is $4-10$ newtons.

Table 6 shows the values of the disintegration time of the 2 batches of tablets produced. The data shows that tablets produced using LC-MCC as binders have a greater disintegration time compared with the tablets produced without binder. This is consistent with the hardness of the tablets produced; the more fragile tablets disintegrated faster.

Results of the statistical analysis used in Table 7 show that the mean disintegration time of the MCC tablets has a significant difference in the mean disintegration time of the tablets produced without binder. The $P$ value of 0.0002 is less than the confidence level of 0.05 ; thus the data has a significant difference. The test statistic value of 12.86 is also greater than the critical value of $t$ (2.776) which also indicates that there is a significant difference in the mean hardness of the two types of tablets. However, based on standards given by
TABLE 7: Data for $t$-test for independent means (disintegration time).

\begin{tabular}{lcc}
\hline & MCC & NB \\
\hline Mean & 32.91 & 2.21 \\
Variance & 9.5129333 & 7.5920333 \\
Observations & 3 & 3 \\
Pooled variance & 8.5524833 & \\
Hypothesized mean difference & 0 & \\
df & 4 & \\
$t$ stat & $\mathbf{1 2 . 8 5 7}$ & \\
$P(T<=t)$ one-tail & 0.0001055 & \\
$t$ critical one-tail & 2.1318468 & \\
$P(T<=t)$ two-tail & $\mathbf{0 . 0 0 0 2}$ & \\
$t$ critical two-tail & $\mathbf{2 . 7 7 6}$ & \\
$t$-test: two-sample test assuming equal variances. &
\end{tabular}

the United States pharmacopeia, the limit for disintegration time for tablets is 30 minutes; thus there is a need for the addition of another excipient such as disintegrants.

\section{Conclusions}

(i) The percentage yield of microcrystalline cellulose produced from Luffa cylindrica fiber indicates that it is a good source of cellulose since it has a relatively high percentage yield.

(ii) The values of the powder properties of LC-MCC produced show fair and passable flow properties and are within the specifications of a powdered pharmaceutical excipient.

(iii) The acetaminophen tablets formulated using LCMCC as binder have a greater hardness and disintegration time compared with the acetaminophen tablets compressed without binder. The mean tablet hardness and disintegration time of the LC-MCC tablets have a significant difference in the mean tablet hardness and disintegration time of the tablets without binder; thus the MCC produced improved the suitability of acetaminophen in the dry compression process. The tablet properties of the tablets produced using LC-MCC as binder do not conform to the specifications of the US pharmacopeia; thus the study of additional methods and excipients is recommended.

\section{Conflict of Interests}

The authors declare that there is no conflict of interests regarding the publication of this paper.

\section{Acknowledgment}

The authors would like to thank Mr. Allen A. Espinosa of the Faculty of Science, Technology and Mathematics of the Philippine Normal University for his untiring guidance and assistance in publishing this paper. 


\section{References}

[1] P. M. Ejikeme, "Investigation of the physicochemical properties of microcrystalline cellulose from agricultural wastes I: orange mesocarp," Cellulose, vol. 15, no. 1, pp. 141-147, 2008.

[2] F. O. Ohwoavworhua and T. A. Adelakun, "Some physical characteristics of microcrystalline cellulose obtained from raw cotton of Cochlospermum planchonii," Tropical Journal of Pharmaceutical Research, vol. 4, no. 2, pp. 501-507, 2005.

[3] I. J. Ogaji, E. I. Nep, and J. D. Audu-Peter, "Advances in natural polymers as pharmaceutical excipients," Pharmaceutica Analytica Acta, vol. 3, article 146, 2012.

[4] A. Ilindra and J. D. Dhake, "Microcrystalline cellulose from bagasse and rice straw," Indian Journal of Chemical Technology, vol. 15, no. 5, pp. 497-499, 2008.

[5] P. Shailendra, S. Shikha, and L. B. Singh, "Natural binding agents in tablet formulation," International Journal of Pharmaceutical \& Biological, vol. 3, supplement 3, pp. 466-473, 2012.

[6] N. B. Gaikwad and G. Kulkarni, "Evaluation of Buchanania cochinchinesis gum as a binder in formulation development of paracetamol tablets," International Journal of Pharmacy and Pharmaceutical Sciences, vol. 5, supplement 2, pp. 283-289, 2013.

[7] V. Chalapathi, T. V. Yuvaraj, and A. Jaganathan, "Formulation of paracetamol tablets using a novel binder isolated from Manihot esculenta.L and its evaluation," International Journal of ChemTech Research, vol. 2, no. 1, pp. 406-411, 2010.

[8] H. Musa, S. N. Ochu, and P. G. Bhatia, "Evaluation of the tablet binding properties of barley (HordeumVulgare) starch," International Journal of Applied Pharmaceutics, vol. 2, no. 4, pp. 4-7, 2010.

[9] G. Siqueira, J. Bras, and A. Dufresne, "Luffa cylindrica as a lignocellulosic source of fiber, microfibrillated cellulose, and cellulose nanocrystals," BioResources, vol. 5, no. 2, pp. 727-740, 2010.

[10] K. D. Arunachalam, P. Velmurugan, and R. R. Balaji, "Antiinflammatory and cytotoxic effects of extract from Plumbago zeylanica," African Journal of Microbiology Research, vol. 4, no. 12, pp. 1239-1245, 2010.

[11] Indumathy, S. Kumar, K. Pallavi, and G. S. Devi, "Antimicrobial activity of whole plant of Luffa cylindrica (Linn) against some common pathogenic micro-organisms," International Journal of Pharmaceutical Sciences and Drug Research, vol. 3, no. 1, pp. 2931, 2011.

[12] G. P. Yoganandam, K. Ilango, K. Sunil, and A. Elumalai, "In vitro antioxidant activity of Luffa cylindrica seed oil," Journal of Global Pharma Technology, vol. 2, no. 3, pp. 93-97, 2009.

[13] P. Muthumani, R. Meera, S. Mary et al., "Phytochemical screening and anti inflammatory, bronchodilator and antimicrobial activities of the seeds of Luffa cylindrica," Research Journal of Pharmaceutical, Biological and Chemical Sciences, vol. 1, no. 4, pp. 11-22, 2010.

[14] A. I. Bamgboye and O. O. Oniya, "Fuel properties of loofah (Luffa cylindrica L.) biofuel blended with diesel," African Journal of Environmental Science and Technology, vol. 6, no. 9, pp. 346$352,2012$.

[15] F. O. Ohwoavworhua and T. A. Adelakun, "Some physical characteristics of microcrystalline cellulose obtained from raw cotton of Cochlospermum planchonii," Tropical Journal of Pharmaceutical Research, vol. 4, no. 2, pp. 501-507, 2005.

[16] Y. J. Oyeniyi and O. A. Itiola, "The physicochemical characteristic of microcrystalline cellulose, derived from sawdust, agricultural waste products," International Journal of Pharmacy and Pharmaceutical Sciences, vol. 4, no. 1, pp. 197-200, 2012.

[17] C. P. Azubuike and A. O. Okhamafe, "Physicochemical, spectroscopic and thermal properties of microcrystalline cellulose derived from corn cobs," International Journal of Recycling of Organic Waste in Agriculture, vol. 1, article 9, 2012.

[18] M. D. O. Bastos, R. B. Friedrich, and R. C. R. Beck, "Effects of filler-binders and lubricants on physicochemical properties of tablets obtained by direct compression: a $2^{2}$ factorial design," Latin American Journal of Pharmacy, vol. 27, supplement 4, pp. 578-583, 2008.

[19] K. P. R. Chowdary, L. S. Danki, and S. N. Hiremath, "Comparative evaluation of binders and disintegrants by formulating lomefloxacin tablets," Der Pharmacia Lettre, vol. 2, supplement 2, pp. 231-236, 2010. 

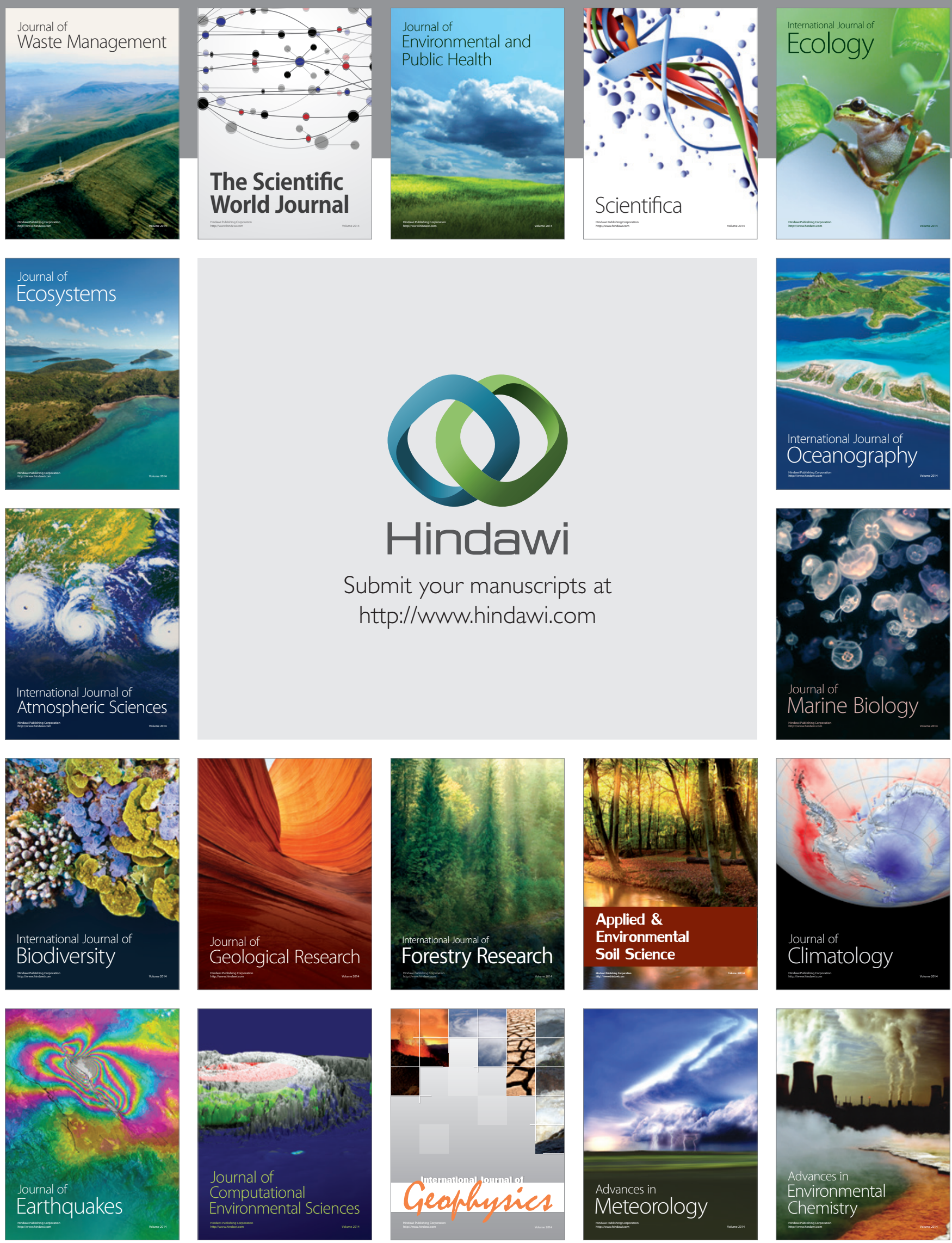